\title{
Factores de riesgo cardiovascular en estudiantes universitarios
}

\author{
Cardiovascular risk factors \\ of college students
}

\section{ABSTRACT}

College students are in a key life stage for the adoption of lifestyles that will be practiced in the family, society and work. During this period, students gain greater autonomy and take responsibility for their own care. University time becomes a critical step in the development of lifestyles. There is a high prevalence of risk factors for non-communicable diseases in university students such as pre-hypertension especially in men and hypercholesterolemia in women. Other risk factors include smoking, physical inactivity, overweight, obesity, low fruit and vegetable consumption, and high consumption of saturated fats. Some differences of cardiovascular risk factors have been found according to gender, year of career and faculty where students attend. It is recommended to consider these differences when we design and perform educational interventions to achieve greater assertiveness and effectiveness. This article reviews the evidence about cardiovascular risk factors in university students according to gender, year of career and faculty where students attend, following the model proposed by Cecchini et al and according to the Framingham study.

Key words: Universitary students, risk factors; chronic diseases; food habits; education.
Gladys Morales I. $(1,2)$

Carlos del Valle R. (3)

Álvaro Soto V. (4)

Daniza Ivanovic M. (5)

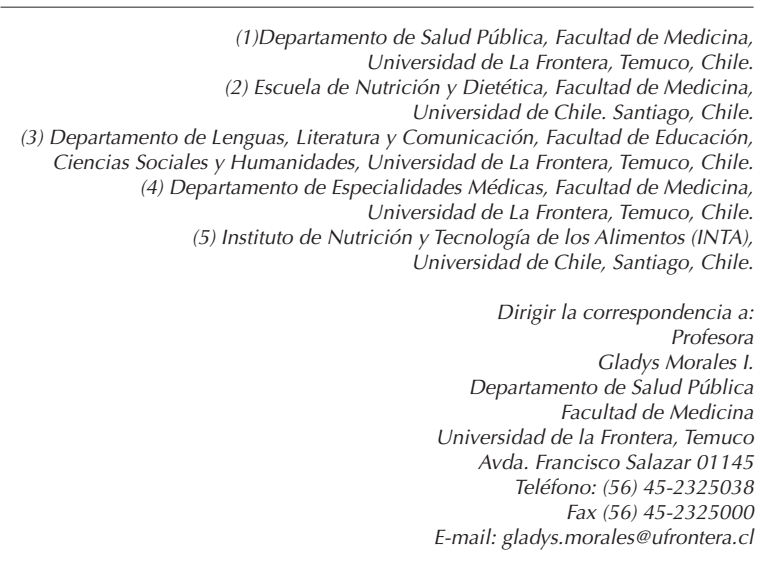

Este trabajo fue recibido el 22 de Mayo de 2012, aceptado con modificaciones el 20 de septiembre de 2012 y aceptado para ser publicado el 10 de Junio de 2013.
INTRODUCCIÓN

Las enfermedades cardiovasculares constituyen un problema de salud pública por su alta prevalencia y por ser la principal causa de muerte de la población adulta en la mayoría de los países (1).

Según diversos estudios, la población de adultos jóvenes parece encaminarse hacia la adopción de conductas de riesgo y estilos de vida poco saludables. Algunos autores lo atribuyen a las débiles conexiones entre la población universitaria con la redes de apoyo; como la familia y los centros educacionales $(2,3)$.

Los estudiantes son sometidos a condiciones particulares al ingresar al sistema universitario. El ambiente académico puede producir cambios en sus estilos de vida, con implicaciones positivas y negativas. Se encuentran en una etapa crítica para el desarrollo de sus hábitos alimentarios, caracterizados por presentar poco tiempo para comer, saltarse comidas frecuentemente, comer entre horas, alto consumo de comida rápida, entre otros $(4,5)$. Sumado a esto, presentan una disminución de la práctica de actividad física por el auge que han tenido los entretenimientos pasivos. Además se ha registrado un incremento en las prevalencias del consumo de tabaco y alcohol $(6,7)$. Estos estilos de vida poco saludables, están contribuyendo al desarrollo de factores de riesgo cardiovascular en los estudiantes universitarios.

La presente revisión bibliográfica tiene por objetivo analizar los factores de riesgo cardiovascular en los estudiantes universitarios según el género, curso y facultad, de acuerdo al Modelo de Prevención de Enfermedades Crónicas (Modelo PEC) de Cecchini y cols. 2010 (8) y al estudio de Framingham (9).

Esperamos que esta revisión sea de utilidad para orientar a quienes diseñan programas de intervención en promoción y prevención en salud para estudiantes universitarios.

Modelo de prevención de enfermedades crónicas (Modelo PEC)

La Organización para la Cooperación y Desarrollo Económico (OECD) y la Organización Mundial de la Salud (OMS) han desarrollado un modelo llamado "Esquema causal para 
los factores de riesgo y enfermedades implementadas en el Modelo PEC". Este modelo ha sido inicialmente aplicado en Europa por un grupo de expertos de la OECD (10).

El modelo explicita tres grupos de enfermedades crónicas: ataque cerebrovascular, cardiopatía coronaria y cáncer (colorrectal, pulmón y de mama). Además define tres grupos de factores de riesgo: proximales, intermedios y distales.

Los factores de riesgo proximales, son aquellos que tienen un efecto directo en la probabilidad de desarrollar cáncer, cardiopatía coronaria y ataque cerebrovascular. Aquí se encuentran, la hipertensión arterial, hipercolesterolemia e hiperglicemia en ayuno.

Los factores de riesgo distales son aquellos que tienen un efecto indirecto en las enfermedades crónicas, aquí se encuentran: el bajo consumo de fibra, alto consumo de grasas y el sedentarismo,

Los factores intermedios son aquellos que están mediados parcialmente por el Índice de Masa Corporal (IMC), actuando sobre los factores de riesgo proximal y en forma directa sobre los eventos de enfermedad.

En la presente revisión se ha decidido incluir también al tabaquismo como un factor de riesgo proximal, ya que de acuerdo al estudio de Framingham, el tabaquismo aumenta el riesgo de infarto agudo al miocardio y muerte súbita (11). Sumado a esto, se ha demostrado que es un factor de riesgo independiente para enfermedades cardiovasculares que puede modificar el perfil lipídico en adultos jóvenes e incrementar el riesgo, especialmente en varones (12). Por otra parte, el tabaquismo es un factor de riesgo conocido para enfermedad cerebrovascular (13).

\section{FACTORES DE RIESGO DISTALES: HABITOS ALIMENTARIOS INADECUADOS Y SEDENTARISMO Consumo de fibra}

En un estudio realizado en Estados Unidos, los estudiantes describieron dificultades en la adopción de comportamientos saludables relacionados con la vida universitaria y señalaron que es un desafío consumir una dieta saludable en la universidad. Por esta razón es necesario entender la transición de comportamientos y desarrollar estilos de vida saludable durante la vida universitaria, considerando la variable género (14).

En diversos países se ha reportado un bajo consumo de fibra en los universitarios (< 25g/día), entre ellos EEUU con 19,9g/día(15), España con menos de 20 g/día en (16), Grecia con 15,9 g/día (17) Irán con 14 g/ día (18). Una situación muy diferente se encontró en universitarios croatas, con 28,9 g y $25,4 \mathrm{~g}$ en hombres y mujeres, respectivamente (19). Con respecto a las prevalencias de consumo de frutas y verduras, de acuerdo a la recomendación " 5 al día", que consiste en el consumo de al menos 5 porciones de frutas y verduras al día. En universitarios colombianos de la carrera de Medicina, tanto en el primer como en el quinto año, sólo el 25,2 \% cumple con la recomendación (20). En Chile, las prevalencias son menores, de acuerdo a la Encuesta Nacional de Salud (ENS) 2009-2010, sólo 11,1 \% y 15,5\% de hombres y mujeres de 15-24 años, respectivamente cumplen la recomendación (21).

\section{Consumo de grasas}

Se ha encontrado un alto consumo de grasas en los estudiantes universitarios. Esto ha sido reportado en países tales como: Grecia (17), Croacia (19), e Irán (18). En este último, se encontró el $50 \%$ de los estudiantes consume 2 o más veces a la semana comida chatarra y, en relación al consumo diario de grasas, se encontró que el $75 \%$ consume aceites vegetales hidrogenados y $56 \%$ consume todos los días mayonesa y salsas saladas altas en grasas (18).

De acuerdo al género, en universitarios chilenos se ha encontrado un mayor consumo de grasas en hombres que en mujeres. La preferencia de los hombres fue la comida chatarra y para las mujeres las golosinas (22). Esto es concordante con lo reportado en universitarios de Palestina, donde existe un $62,9 \%$ de hombres que consume comida chatarra 3 veces a la semana y mujeres con un consumo de $37,1 \%$ (23).

En relación al curso, se ha encontrado que los hombres de tercer año presentan un mayor consumo de grasas y bocadillos (papas fritas, ramitas), galletas, completos y bebidas, alimentos de alta densidad energética, ricos en grasa saturada, azúcar y sal, que los estudiantes de igual género de primer año (24).

Con respecto al área científica o facultad a la que pertenecen los estudiantes, es muy escasa la evidencia existente. Tirodimos y cols. 2009, reportaron que no se presentaron diferencias significativas entre las facultades de acuerdo a los hábitos alimentarios (25).

\section{Sedentarismo}

De acuerdo a diversos estudios, se ha constatado que uno de los factores de riesgo más frecuentes entre los estudiantes universitarios es el sedentarismo. En universitarios de los Emiratos Árabes se ha reportado prevalencias de 73\% (26), en Venezuela un $72,5 \%$ (27). Sin embargo, en Chile se han reportado prevalencias de $87,8 \%(28)$ y $75,9 \%$ según la ENS 2009-2010 (para el grupo etario de 15- 24 años) (21).

Con respecto al género, se ha encontrado que las mujeres universitarias son más sedentarias, que los hombres. De acuerdo al estudio realizado por Martínez y cols. 2012, en estudiantes chilenos de la Universidad Austral de Chile, se encontró un $91 \%$ de sedentarismo en mujeres y un $81 \%$ en hombres (28).

En relación a la práctica de actividad física de acuerdo al curso al que pertenecen los universitarios portugueses, se encontró que los estudiantes de primer año, y los de cursos superiores, presentan una alta prevalencia de sedentarismo (79,6\% y $80,7 \%$ en hombres y mujeres, respectivamente) (29). Otro estudio realizado por Martínez y cols. 2009, encontraron en universitarios españoles una prevalencia de sedentarismo de $37 \%$ y $41,2 \%$, en mujeres y hombres respectivamente (30). En estudiantes colombianos de medicina de quinto año hubo diferencias en comparación con los de primero (45,5\% y 25,9\% respectivamente) (20). Estas diferencias pudieran explicarse por el período de aplicación de las encuestas (al inicio o término del semestre), debido a que se ha evidenciado que la práctica de ejercicio físico aeróbico disminuye significativamente entre el inicio y el final del primer año de universidad (4).

Con respecto a la facultad o área científica de estudio, de acuerdo a Brandao y cols, 2008, se encontró una mayor prevalencia de sedentarismo en el área de Ciencias Sociales y Humanas $(66,6 \%)$ en comparación con sus pares de las otras áreas (31).

Es importante mencionar que la Estrategia Nacional de Salud 2011-2020 en Chile, tiene como una de sus metas, lograr un 30\% de jóvenes físicamente activos al año 2020 (32).

\section{SOBREPESO Y OBESIDAD: FACTORES DE RIESGO INTERMEDIO}

En nuestra población de universitarios, la transición desde la enseñanza media a la universidad representa un cambio importante en su estilo de vida, constituyendo un período crítico de riesgo para el incremento de peso. En nuestro país, 
de acuerdo a la ENS 2009-2010 se encontró un IMC promedio de $24,4 \mathrm{~kg} / \mathrm{m} 2$ tanto en hombres como en mujeres de $15-24$ años (21), muy cercano al límite superior de la normalidad (IMC normal: 18,5-24,9 kg/m2). En estudiantes universitarios de EEUU, se ha evidenciado un incremento significativo del IMC, porcentaje de grasa corporal y circunferencias de cintura y cadera, evaluados antes y durante el primer año de universidad $(4,33)$.

De acuerdo a Deliens y cols 2013, que investigaron a los universitarios belgas durante el primer semestre de estadía en la universidad, encontraron que el vivir en residencias estudiantiles se relaciona con un incremento en el porcentaje de grasa corporal. Esto podría explicarse por cambios en el estilo de vida: hábitos alimentarios inadecuados y aumento de la inactividad física, debido a la transición de vivir bajo el control de los padres a vivir en forma independiente (34).

Se ha encontrado que los estudiantes de primer año presentan un estado nutricional más adecuado en comparación con los estudiantes de cursos superiores. (24). Esta situación ha sido demostrada en otros estudios, en los cuales se observa una tendencia al aumento del IMC por año de estudio. Además, se ha reportado que el primer año de universidad es crítico en la ganancia de peso $(35,36)$. Sin embargo, de acuerdo a un meta-análisis realizado por Vella- Zarb y Elgar 2009 , se llegó a la conclusión que el promedio de peso ganado durante el primer año de universidad es de 1,75 kg (37).

Por otra parte, se ha evidenciado que existen diferencias significativas entre los estudiantes que inician su primer año con sobrepeso y obesidad, porque tienen mayor riesgo de ganar peso, en comparación con los que presentan un estado nutricional normal. Por lo tanto, se recomienda intervenir a los estudiantes con sobrepeso y obesidad desde que ingresan a la universidad (38).

Se ha demostrado que las mujeres universitarias son las que presentan las menores prevalencias de sobrepeso y obesidad (IMC > $25 \mathrm{~kg} / \mathrm{m} 2)$, en comparación con los hombres. Esto ha sido observado en países tales como: Libania (39), Grecia (25), Suiza (40) y Chile (41). Otros estudios realizados en Corea (42), España (43), Serbia (44), Brasil $(45)$ y Chile $(24,46)$, han reportado que el IMC promedio tanto en hombres como en mujeres se ha presentado dentro de los rangos normales, observándose los promedios de IMC más bajos en las mujeres en comparación con los hombres. Sin embargo, en algunos de estos estudios, el porcentaje de grasa corporal promedio de las mujeres, se ha encontrado en el límite superior de la normalidad $(24,45)$.

Con respecto al área científica de estudio, son bastantes escasos los estudios que comparen las prevalencias de obesidad. De acuerdo al estudio realizado por Brandao y cols., en estudiantes portugueses, no se encontraron diferencias significativas en las prevalencias de obesidad entre las diversas áreas, correspondientes a 3,6\% en Ciencias sociales y Humanas, 3,3\% en Ciencias Naturales y Técnicas y de 2,9\% en Ciencias de la Salud (31).

\section{FACTORES DE RIESGO PROXIMALES: HIPERTENSION ARTERIAL, HIPERGLICEMIA, HIPERCOLESTEROLEMIA Y TABAQUISMO Hipertensión arterial}

En los diversos estudios revisados, se clasificó a estudiantes con pre-hipertensión arterial (pre- HTA) a los que presentaban una presión arterial sistólica (PAS) entre 120-139 $\mathrm{mmHg}$ o una presión arterial diastólica (PAD) entre 80-89 mm $\mathrm{Hg}$. La hipertensión arterial ha sido definida en estudiantes que presenten una $P A S \geq$ a $140 \mathrm{mmHg}$ ó $P A D \geq 90 \mathrm{mmHg}$. Se ha encontrado una alta prevalencia de pre-HTA en Chile, especialmente en hombres en comparación con las mujeres (48,2\% v/s 27,5\%) (28). Resultados más bajos se han reportado en universitarios palestinos. (38\% v/s 11,2 \%) (23) La prevalencia de HTA ha sido determinada en 13,7\% y 3,5\% en Portugal (29), $8,5 \%$ y $2,0 \%$ en Chile (28) y un 3,3\% y $0,4 \%$ en Palestina en hombres y mujeres, respectivamente (23).

En estudiantes coreanos, se han encontrado altos promedios de presión arterial sistólica en hombres en comparación con las mujeres $(128,9 \mathrm{~mm} / \mathrm{Hg}$ y $109,8 \mathrm{~mm} / \mathrm{Hg}$, respectivamente). Sin embargo la presión arterial diastólica promedio se encontró dentro de rangos normales (42).

Con respecto al curso, Brandao y cols, 2011 reportaron que estudiantes portugueses de primer año no presentaban hipertensión arterial en comparación con un 1,4\% que presentaron los estudiantes con más de un año de estadía en la universidad (29). De acuerdo al área científica a la que pertenecían los universitarios, según Brandao y cols. 2008, se observó que la menor prevalencia de hipertensión arterial era en Ciencias de la Salud en comparación con las otras facultades o áreas científicas (31).

\section{Hiperglicemia}

Con respecto a la hiperglicemia (glicemia en ayunas $\geq$ a $100 \mathrm{mg} / \mathrm{dL}$ ), se ha detectado en universitarios chilenos un $2,1 \%$ (28), $1,3 \%$ (47) y $1,4 \%$ (glicemias entre $101-125 \mathrm{mg} / \mathrm{dL}$ ) (48). Esto es concordante con otro estudio realizado en Chile en población adulta de 18 a 24 años, en el cual se encontró una prevalencia de $2,4 \%$ de hiperglicemia (49). En relación a glicemias $\geq$ a 126 mg/dL, de acuerdo a la ENS 2009-2010, en población de 15-24 años, se encontró una baja prevalencia en ambos géneros $(0,2 \%$ en hombres y $0,6 \%$ en mujeres) (21). Esto es concordante con los valores observados en universitarios de Portugal, pero con diferencias significativas por género con respecto a la hiperglicemia (glicemia en ayunas $\geq$ a $109 \mathrm{mg} / \mathrm{dL}$ ). Los hombres no presentaron hiperglicemia, en comparación con las mujeres que presentaron un 1,2\% (31).

Los estudiantes portugueses de primer año, no presentaron hiperglicemia en comparación con un $0,9 \%$ de prevalencia en cursos superiores (29). Situación similar se encontró al comparar las diversas áreas científicas a las que pertenecían los estudiantes, con prevalencias de hiperglicemia fueron 1,1\% para Ciencias Naturales y Técnicas, 1,0\% para Ciencias de la Salud y 0\% para Ciencias Sociales (31).

\section{Hipercolesterolemia}

Se ha encontrado una mayor prevalencia de colesterol total (CT) elevado ( $\geq 200 \mathrm{mg} / \mathrm{dl}$ ) en universitarios de nuestro país de sexo femenino, comparado con hombres, correspondientes a $18,4 \%$ y $10,6 \%$, respectivamente (28). Esto es concordante con lo reportado en estudiantes portugueses, con prevalencias de $23,2 \%$ y $6,4 \%$ en mujeres y hombres, respectivamente (31). El valor de CT promedio en estudiantes de Brasil, se evidenció que las mujeres presentan mayor valor $(168,8 \mathrm{mg} / \mathrm{dl})$, en comparación con los hombres (141,4 mg/dL) (45).

Con respecto al colesterol LDL elevado (cLDL $\geq 130 \mathrm{mg} /$ $\mathrm{dL}$ ) no existen diferencias significativas por género. En universitarios portugueses se ha reportado una prevalencia de $7,4 \%$ (31). En Chile, se ha observado mayores prevalencias, 20,9\% en mujeres y de 14,9 en hombres (28). Con respecto a los valores promedios de cLDL, de acuerdo a la ENS 2009-2010 (para el grupo etario de 15- 24 años), se encontró $89,1 \mathrm{mg} / \mathrm{dL}$ y $93,9 \mathrm{mg} / \mathrm{dL}$ en hombres y mujeres, respectivamente (21). En 
países árabes, se ha reportado un valor promedio de CLDL de 109,8 mg/dl en hombres y mujeres (26), muy similar a Japón (valor promedio de $106,1 \mathrm{mg} / \mathrm{dL}$ ) (50).

Finalmente, es importante mencionar una mayor prevalencia de dislipidemia (CT > $190 \mathrm{mg} / \mathrm{dL}$ o LDL> 130 o HDL < 40 y TG > $150 \mathrm{mg} / \mathrm{dL}$ ) en estudiantes portugueses que han cursado algunos años de universidad en comparación con los de primer año, con $44 \%$ y $28,6 \%$, respectivamente (29). De acuerdo a la facultad ó área científica a la que pertenecían los estudiantes, se encontró que el área de las Ciencias de la Salud presentó la mayor prevalencia de hipercolesterolemia, en comparación con el área de Ciencias Naturales y Técnicas (20,2\% y 13,7 , respectivamente) (31).

Tabaquismo

En universitarios de Grecia, se han reportado tabaquismo en $28.4 \%$ y $33.2 \%$ (51), en Venezuela de $27,9 \%$ y $42,3 \%$ (27) y en Portugal $4,7 \%$ y $14,5 \%$ para mujeres y hombres, respectivamente (31).

En universitarios chilenos se reportan altas prevalencias de estudiantes fumadores actuales (fumadores en forma diaria y ocasionales), sin diferencias significativas por género, con un $40,1 \%$ y $39,2 \%$ (49), $30 \%$ y $35,8 \%$ (52), $28,7 \%$ y $27,7 \%$ (28), en mujeres y hombres, respectivamente. De acuerdo a la ENS 2009-2010 (para el grupo etario de 15- 24 años), se han reportado prevalencias, de $42,6 \%$ y $48,3 \%$ en mujeres y hombres, respectivamente (21).

Se ha reportado que los estudiantes de primer año de la universidad presentan un menor consumo de tabaco y alcohol que los estudiantes de cursos superiores, en varios países tales como: Chile $(24,52)$, Portugal (29), Colombia (20) y España (30).

Si se considera el área de estudio de los estudiantes, existen estudios que demuestran diferencias significativas por facultad. Según Morales y cols. 2012, una menor prevalencia de consumo de tabaco en la facultad del área de salud (17\%), en comparación con otras facultades y, la mayor prevalencia se observó en el área de Educación y Ciencias Sociales con un $41,7 \%$ (52); concordante con lo encontrado por Brandao y cols. 2008 y Tirodimos et al 2009, que reportaron que los estudiantes de la Facultad de Medicina o área de Ciencias de la Salud presentan las menores prevalencias en comparación con las otras facultades $(31,25)$.

Lo anteriormente mencionado, podría explicarse porque los estudiantes que postulan a carreras relacionadas con esta área pudieran tener un mayor conocimiento e interés por estilos de vida saludables, en comparación con los estudiantes de otras carreras (52).

\section{CONCLUSIONES}

1. De acuerdo a los factores de riesgo cardiovascular analizados, se puede concluir que los estudiantes universitarios se encuentran en un período crítico, propensos a enfermedades crónicas no transmisibles.

2. Se encontró en la categorización de factores de riesgo distales un bajo consumo de fibra, de frutas y verduras y un alto consumo de grasas (especialmente en hombres); una alta prevalencia de sedentarismo, especialmente en mujeres.

3. En relación a los factores de riesgo intermedio (sobrepeso y obesidad), es importante mencionar que el IMC, porcentaje de grasa corporal y circunferencias de cintura y cadera, se incrementan significativamente durante el primer año de estadía en la universidad. Los estudiantes de cursos superiores presentan una mayor prevalencia de sobrepeso y obesidad, en comparación con el primer año. Se recomienda considerar en futuros estudios tanto el IMC, como la medición del porcentaje de grasa corporal, para un diagnóstico nutricional más certero.

4. Respecto a los factores de riesgo proximales, destaca una alta prevalencia de pre-HTA en los universitarios, especialmente en hombres. En relación al CT elevado, las mujeres tienen una mayor prevalencia. Es importante considerar, que las mayores prevalencias de dislipidemias se detecten en estudiantes de cursos superiores en comparación con los de primer año.

5. Se han encontrado bajas prevalencias de hiperglicemia en ayunas en los estudiantes universitarios. Por lo tanto, para futuros estudios se recomienda considerar la insulinemia, aparte de la glicemia en ayunas, para detectar alteraciones más precoces del metabolismo de la glucosa.

6. El tabaquismo, es una situación preocupante, debido a alto consumo de tabaco en estudiantes universitarios, con diferencias entre las distintas facultades y por curso, destacando que existe un mayor consumo en cursos superiores en comparación con el primer año de estudio. Por lo tanto nos parece recomendable la realización de programas educativos de prevención del consumo de tabaco en los primeros años de universidad.

7. Se recomienda seguir realizando estudios sobre los factores de riesgo cardiovascular en universitarios, diferenciados por curso, género y facultad, debido a que son muy escasos los estudios existentes. Además para quienes diseñan programas de intervención en promoción y prevención en salud, se recomienda que ellos comiencen desde el primer año de estudio, entregando las herramientas necesarias para evitar el sobrepeso y la obesidad, considerando las diferencias por curso, género y facultad para enfrentar los factores de riesgo que están afectando a los universitarios. Este enfoque diferenciado podría permitir una mayor asertividad y efectividad en las intervenciones.

\section{RESUMEN}

Los estudiantes universitarios se encuentran en una etapa del ciclo vital clave para la adopción de estilos de vida, que practicarán en el ámbito familiar, social y laboral. En este periodo los estudiantes adquieren mayor autonomía y asumen la responsabilidad de su autocuidado, por lo cual se convierte en una etapa crítica para el desarrollo de sus estilos de vida. Existe una alta prevalencia de factores de riesgo de enfermedades crónicas no transmisibles en los universitarios, entre los que destacan pre-hipertensión especialmente en hombres y la hipercolesterolemia en mujeres. También el tabaquismo, sedentarismo, sobrepeso, obesidad, bajo consumo de frutas y verduras, y alto consumo de grasas saturadas. Se encontraron diferencias en los factores de riesgo cardiovascular de acuerdo al género, curso y facultad de los estudiantes. Se recomienda consideran estas diferencias al diseñar y realizar intervenciones educativas para lograr una mayor asertividad y efectividad. El presente artículo revisa la evidencia sobre los factores de riesgo cardiovascular en estudiantes universitarios de acuerdo al género, curso y facultad, según el modelo propuesto por Cecchini y cols. y al estudio de Framingham.

Palabras clave: Estudiantes universitarios; factores de riesgo; enfermedades crónicas; hábitos alimentarios; educación.

\section{BIBLIOGRAFÍA}

1. WHO. World Health Organization. The World Health Report 2002. Reducing Risks, Promoting Healthy Life. WHO 
2002. Disponible en: http://www.who.int/whr/2002/en/ whrO2_en.pdf

2. Eaton DK, Kann L, Okoro CA and Collins J. Selected Health status indicators and behaviors of young adults, United States 2003. Am J Health Educ 2007; 38: 66-75.

3. Paez M, Castaño J. Estilo de vida y salud en estudiantes de la Facultad de Medicina de la Universidad de Manizales. Arch Med 2009; 9: 146- 64.

4. Pullman AW, Masters RC, Zalot LC, Carde LE, Saraiva MM, Dam YY et al. Effect of the transition from high school to university on anthropometric and lifestyle variables in males. Appl Physiol Nutr Metab. 2009; 34: 162- 71.

5. Barić I, Sataliç Z and Lukešić Ž. Nutritive value of meals, dietary habits and nutritive status in Croatian university students according to gender. Int I Food Sci Nutr 2003; 54: 473- 84

6. López-Azpiazu I, Sánchez-Villegas A, Johansson L, Petkeviciene J, Prättälä R and Martínez-González MA. Disparities in food habits in Europe: systematic review of educational and occupational differences in the intake of fat. J Hum Nutr Diet 2003; 16: 349- 64

7. Hill J, Wyatt $H$, Reed $G$ and Peters JC. Obesity and the environment where do we go from here?. Science 2003; 299:853-55.

8. Cecchini M, Sassi F, Lauer J, Lee Y, Guajardo-Barrón V and Chisholm D. Tackling of unhealthy diets, physical inactivity, and obesity: health effects and cost- effectiveness. Lancet. 2010; 376:1775- 84 .

9. Anderson KM, Wilson PWF, Odel PM and Kannel WB. An update coronary risk profile. A statement for health professionals. Circulation 1991, 83:356-62.

10. Sassi F, Cecchini M, Lauer J and Chisholm D. Improving lifestyles, tackling obesity: the health and economic impact of prevention strategies. OECD Health working paper 48. Paris: OECD, 2009. Available from: http://www.oecdilibrary.org/docserver/download/5ks5pqlc5jnn.pdf?expir es $=1361632617 \& i d=i d \& a c c n a m e=$ guest\&checksum $=556$ D20987D76FOBA13579420BD7C7A6C.

11. Doyle JT, Dawber TR, Kannel WB, Heslin AS and Kahn HA. Cigarette smoking and coronary heart disease: combined experience of the Albany and Framingham studies. N Engl J Med. 1962; 266: 796-801.

12. Maćkowiak K, Nowicki M, Wysocka E, Brozek $A$ and Torliński L.The impact of tobacco smoking on the selected risk factors for cardiovascular disease in students of Poznan University of Medical Sciences. Przeg/ Lek. 2012; 69: 819- 23.

13. O'Donnell MJ, Xavier D, Liu L, Zhang H, Chin SL, RaoMelacini $P$, et al. Risk factors for ischaemic and intracerebral haemorrhagic stroke in 22 countries (the INTERSTROKE study): a case-control study. Lancet 2010: 376; 112-23.

14. Cluskey M and Grobe D. College weight gain and behavior transitions: male and female differences. J Am Diet Assoc. 2009; 109: 325-9.

15. Byrd-Williams CE, Strother ML, Kelly LA and Huang $T T$. Dietary fiber and associations with adiposity and fasting insulin among college students with plausible dietary reports. Nutrition. 2009; 25: 896-904.

16. Montero A, Úbeda N, García A. Evaluación de los hábitos alimentarios de una población de estudiantes universitarios en relación con sus conocimientos nutricionales. Nutr Hosp. 2006; 21: 466- 73 .

17. Chourdakis M, Tzellos T, Pourzitaki C, Toulis KA, Papazisis $G$ and Kouvelas D. Evaluation of dietary habits and assess- ment of cardiovascular disease risk factors among Greek university students. Appetite 2011; 57: 377-83.

18. Azadbakht L and Esmaillzadeh A. Macro and Micro-Nutrients Intake, Food Groups Consumption and Dietary Habits among Female Students in Isfahan University of Medical Sciences. Iran Red Crescent Med J. 2012; 14: 204- 9.

19. Satalic Z, Baric IC and Keser I. Diet quality in Croatian university students: energy, macronutrient and micronutrient intakes according to gender. Int J Food Sci Nutr. 2007; 8: 398-410.

20. Alba LH. Perfil de riesgo en estudiantes de Medicina de la Pontificia Universidad Javeriana. Univ. Med. Bogotá 2009; 50: 143-55.

21. Encuesta Nacional de Salud ENS Chile 2009-2010 http:// www.redsalud.gov.cl/portal/url/item/99bbf09a908d3eb8 e04001011f014b49.pdf

22. Espinoza L, Rodríguez F, Gálvez J, Mc Millan N. Hábitos alimentarios y de actividad física en universitarios. Rev Chil Nutr. 2011; 38: 458-65.

23. Tayem $Y$, Yaseen $N$, Khader $W$, Abu Rajab L, Ramani $A$ and Saleh M. Prevalence and risk factors of obesity and hypertension among students at a central university in the West Bank. Libyan J Med 2012. Available from: http:// dx.doi.org/10.3402/ljm.v7i0.19222.

24. Durán S, Castillo M, Vío F. Diferencias en la calidad de vida de estudiantes universitarios de diferente año de ingreso del campus Antumapu. Rev Chil Nutr 2009; 36 :200-9.

25. Tirodimos I, Georgouvia I, Savvala TN, Karanika E and Noukari D. Healthy lifestyle habits among Greek university students: differences by sex and faculty of study. East Mediterr Health J, 2009; 5:722-8.

26. Khan N, Gomathi KG, Shehnaz SI and Muttappallymyalil J. Diabetes Mellitus-related knowledge among University Students in Ajman, United Arab Emirates. Sultan Qaboos Univ Med J. 2012; 12: 306- 14.

27. Oviedo G, Morón de Salim A, Santos I, Sequera S, Soufrontt $G$, Suárez $P$ et al. Factores de riesgo de enfermedades crónicas no transmisibles en estudiantes de la carrera de Medicina. Universidad de Carabobo, Venezuela. Año 2006. Nutr Hosp. 2008; 23: 288- 93.

28. Martínez $M$, Leiva A, Sotomayor C, Victoriano $T$, Von Chrismar A, Pineda S. Factores de riesgo cardiovascular en estudiantes de la Universidad Austral de Chile. Rev Med Chil. 2012; 140: 426- 35.

29. Brandão MP, Pimentel FL and Fonseca M. Impact of academic exposure on health status of university students. Rev Saude Publica 2011;45: 49- 58.

30. Martínez A, Balanza S, Leal M, Martínez A, Conesa C, Abellán J. Relación entre el consumo de tabaco y alcohol y el ejercicio físico con el paso por la universidad. Aten Primaria.2009; 41: 558- 63.

31. Brandaõ MP, Pimentel FL, Silva CC and Cardoso MF. Risk factors for cardiovascular disease in a Portuguese university population. Rev Port Cardiol. 2008; 27:29-35.

32. Ministerio de Salud. Metas 2011-2020 Elige Vivir sano. Objetivo Estratégico 3. Factores de riesgo. Págs. 113143. Disponible en: http://www.minsal.gob.cl/portal/ docs/1/5648346.pdf

33. Wengreen $\mathrm{H}$ and Moncur C. Change in diet, physical activity, and body weight among young-adults during the transition from high school to college. Nutr J 2009; 8:32. Available from: http://www.nutritionj.com/content/8/1/32.

34. Deliens T, Clarys P, Van Hecke L, De Bourdeaudhuij L and 
Deforche B. Changes in weight and body composition during the first semester at university. A prospective explanatory study. Appetite. 2013; 65:111-6.

35. Anderson DA, Shapiro JR and Lundgren JD. The freshman year of college as a critical period for weight gain: An initial evaluation. Eat Behav.2003; 4: 363-7.

36. Dart $L$ and Davis M. Vigorous physical activity patterns among college students. TAFCS Res J. 2008; 1: 24-6.

37. Vella-Zarb RA and Elgar FJ. The 'Freshman 5'. A metaanalysis of weight gain in the freshman year of college. $J$ Am Coll Health. 2009; 58:161-6.

38. Kasparek DG, Corwin SJ, Valois RF, Sargent RG and Morris RL. Selected Health Behaviors That Influence College Freshman Weight Change. J Am Coll Health. 2008; 56:4, 437-44.

39. Yahia N, Achkar A, Abdallah A and Rizk S. Eating habits and obesity among Lebanese university students. Nutr J. 7:32. Available from: http://www.nutritionj.com/ content/7/1/32

40. 40.Von Bothmer MI and Fridlun B. Gender differences in health habits and in motivation for a healthy lifestyle among Swedish university students. Nurs Health Sci. 2005; 7:107-18

41. Mc Coll P, Amador M, Aros J, Lastra A, Pizarro C. Prevalencia de factores de riesgo de enfermedades crónicas no transmisibles en estudiantes de medicina de la Universidad de Valparaíso. Rev Chil Pediatr. 2002; 73: 478- 82.

42. Shin N, Hyun W, Lee $H$, Ro M and Song $K$. A study on dietary habits, heath related lifestyle, blood cadmium and lead levels of college students. Nutr Res Pract. 2012; 6: 340-8.

43. Ledo-Varela MT, De Luis DA, González-Sagrado M, Izaola $O$, Conde $R$, Aller $R$. Características nutricionales y estilo de vida en universitarios. Nutr Hosp. 2011; 26: 814-8.

44. Pantic I, Malbasa M, Ristic S, Turjacanin D, Medinica
S, Paunovic J and Pantic S. Screen viewing, body mass index, cigarette smoking and sleep duration in Belgrade University student population: results of an observational, cross-sectional study. Rev Med Chil. 2011; 139: 896-901.

45. Costa Silva ZJ, Barreto CL, De Castro CL, Duarte PG, Toyomi $H A$ and Sachs A. Lipid profile and cardiovascular risk factors among first-year Brazilian university students in São Paulo. Nutr Hosp. 2011; 26: 553-9.

46. Espinoza L, Rodríguez R., Gálvez J, MacMillan N. Hábitos de alimentación y actividad física en estudiantes universitarios. Rev Chil Nutr. 2011, 38: 458- 65.

47. Bustos P, Amigo H, Arteaga A, Acosta AM, Rona RJ. Factores de Riesgo de Enfermedad Cardiovascular en Adultos Jóvenes. Rev Med Chil. 2003; 131: 973-80.

48. Palomo I, Torres G, Alarcón M, Maragaño P, Leiva E, Mujica $V$. Alta prevalencia de factores de riesgo cardiovascular clásicos en una población de estudiantes universitarios de la región centro-sur de Chile. Rev Esp Cardiol. 2006; 59. 1099-105.

49. Palomo I, Icaza G, Mujica V,Núñez L. Leiva E, Vásquez M et al. Prevalencia de factores de riesgo cardiovascular clásicos en población adulta de Talca, Chile, 2005. Rev Med Chil. 2007; 135: 904-12.

50. Michiko E, Nakanishi $Y$ and Miyatake N. The Relation between Insulin Resistance and Lifestyle in Japanese Female University Students. Acta Med. Okayama, 2011; 65:199-204.

51. Mammas IN, Bertsias GK, Linardakis M, Tzanakis NE, Labadarios DN and Kafatos AG. Cigarette smoking, alcohol consumption, and serum lipid profile among medical students in Greece. Eur J Public Health. 2003; 13:278-82.

52. Morales G, Del Valle C, Belmar C, Orellana Y, Soto A, Ivanovic $D$. Prevalencia de consumo de drogas en estudiantes universitarios que cursan primer y cuarto año. Rev Med Chil. 2011; 139: 1573-80. 